\title{
QUANTENPHYSIK
}

\section{Kooperativ oder selbstbezogen - kann ein Elektron wählen?}

Das klassische Doppelspaltexperiment, das Thomas Young $1802 \mathrm{mit}$ Licht ausführte, ist heute in vielen Varianten möglich. Zum Beispiel kann man als „Doppelspalt“ die beiden Atome eines Moleküls verwenden, an denen Elektronen gestreut werden, die das Molekül selbst emittiert. Eine deutsch-amerikanische Forschergruppe unter maßgeblicher Beteiligung des Fritz-Haber-Instituts in Berlin konnte jüngst zeigen, dass das Interferenzverhalten von der Energie der ausgesandten Elektronen abhängt. Ab einer kritischen Energie überlagert sich das gestreute Elektron nicht mehr mit seinem Nachbarelektron, sondern mit sich selbst, wobei es eine Abbildung seiner Umgebung erzeugt [1].

Wellen besitzen neben ihrer Amplitude eine Phase bezüglich derer sie untereinander gekoppelt werden können. Es entsteht eine kohärente Überlagerung der Einzelwellen, bei der sie ihre jeweilige Identität verlieren. Lediglich ihre Summen- und Differenzeigenschaften bleiben erhalten. Dies gilt sowohl für klassische als auch für Materiewellen.

Ein analoges Verhalten zeigen homonukleare zweiatomige Moleküle, wie $\mathrm{N}_{2}$, wenn sie nach der Absorption eines hochenergetischen Photons ein Elektron emittieren. Dieses Elektron kann gleichermaßen von dem einen oder dem anderen Atom des Moleküls emittiert werden, da beide Atome spiegelsymmetrisch und ununterscheidbar sind. Dies wies unsere Gruppe am Fritz-Haber-Institut der Max-Planck-Gesellschaft experimentell vor drei Jahren nach [2].

Die Elektronen tunneln zwischen den beiden Atomen hin und her, wobei sich nicht unterscheiden lässt, von welchem Atom das Photoelektron ursprünglich emittiert worden ist. Es zeigt stattdessen Eigenschaften einer kohärenten Überlagerung zweier Wellen, die von beiden Seiten „gleichzeitig“ emittiert worden sind.

Dieses kohärente, nicht-selbstreferentielle Verhalten bleibt erhalten, wenn das emittierte Elektron am Nachbaratom gestreut wird. Das gestreute Elektron überlagert sich weiter mit der vom Nachbaratom emittierten Elektronenwelle. Wegen des Tunneleffekts wird zeitintegriert gewissermaßen immer ein halbes Elektron von jeder Seite emittiert. Nun überlagert sich die Welle des halben gestreuten Elektrons mit der Welle des halben nichtgestreuten Elektrons vom Nachbaratom, nicht aber mit seiner eigenen, nicht gestreuten Welle. Diese Überlagerung ist jedoch destruktiv, da die Streuung eine Phasenverschiebung um verursacht. Sie verändert damit die Amplitude der ursprünglichen Welle. Ihr kohärenter Charakter bleibt aber grundsätzlich erhalten, die Elektronen bleiben Teil einer kohärenten, nicht-selbstreferentiellen Umgebung. Gilt dieses Prinzip der Kohärenzerhaltung aber für alle Elektronen, den gestreuten und ungestreuten, unabhängig von ihrer jeweiligen Energie? 
Dieser Frage ist unsere Gruppe zusammen mit Kollegen von der Universität Franfurt nachgegangen. Dazu bestrahlten wir im HASYLAB des DESY in Hamburg und bei BESSY in Berlin $\mathrm{N}_{2}$-Moleküle mit monochromatischer Röntgenstrahlung, deren Energie über einen weiten Bereich variiert wurde. Dies führte zur Emission von Photoelektronen mit entsprechend unterschiedlichen kinetischen Energien. Die Intensität dieser Elektronen sollte nun in Abhängigkeit von der jeweiligen kinetischen Energie in einer Weise oszillieren, wie es von einer kohärenten Überlagerung von zwei Wellen zu erwarten wäre, die von zwei räumlich getrennten Orten emittiert werden. Dies sollte sowohl für die ungestreuten als auch für die gestreuten Elektronen gelten.

Überraschenderweise tritt dieses kohärente Verhalten für die gestreuten Elektronen jedoch nur auf, wenn deren Materie- oder De-Broglie-

Wellenlänge größer als der Abstand zwischen den beiden Emitter-Atomen ist. Ist die Materiewellenlänge kleiner als dieser Abstand, so geht die Kohärenz zwischen den beiden Emissionsorten verloren, und die gestreute Welle überlagert sich selbst-referentiell mit ihrer eigenen ungestreuten Welle.

Dieses unerwartete experimentelle Ergebnis konnten anschließend Forscher des California Instituts of Technology in Pasadena, Kalifornien, mit theoretischen Berechnungen bestätigen. Bleibt die Frage, warum es zu dieser Änderung vom kooperativ kohärenten zum selbst-referentiellen Verhalten kommt.

Die Antwort ist das örtliche Auflösungsvermögen der gestreuten Elektronenwelle. So lange die Wellenlänge der gestreuten Welle größer als der Abstand zwischen den beiden Emissionszentren ist, bleibt die ursprüngliche Kohärenz auch bei Streuung erhalten. Sobald jedoch die Materiewellenlänge kleiner als der Abstand zwischen den beiden Atomen wird und das Elektron diesen Abstand im Sinne eines „HeisenbergMikroskops“ räumlich auflösen kann, geht diese Kohärenz verloren. Das nunmehr ortsauflösende Elektron wird phasenunabhängig bezüglich des Nachbaratoms zurückgestreut und überlagert sich anschließend mit seiner eigenen ungestreuten Welle.

Dieser Prozess ist seit vielen Jahrzehnten als Extended X-ray Fine Structure (EXAFS) bekannt, jedoch nur für heteronukleare Moleküle. Für homonukleare Systeme hat man wegen der Spiegelsymmetrie keine Selbstinterferenz erwartet, sondern nur Interferenz mit der jeweiligen Nachbarwelle, die über den Tunneleffekt vom Nachbaratom emittiert worden ist. Die EXAFS-Analyse ist die Grundlage einer der erfolgreichsten Methoden zur Strukturanalyse fester Materie, da das selbst-referentielle Verhalten der gestreuten Photoelektronen detaillierte Informationen über seine Umgebung liefert. In unserem Fall ist es für seine räumlich lokale Umgebung empfindlich, weil es seinen ursprünglich nicht-lokalen, kohärenten Charakter verloren hat und deshalb in der Lage ist, Informationen über seine Umgebung zu sammeln. Es ist im Prinzip die „Welcher-Weg“-Information des Einzelteilchen-Doppelspaltexperiments, die in letzter Konsequenz zu einer holographischen Abbildung ihrer Umgebung führt. 
Damit ist es unserer Kollaboration erstmalig gelungen, den Übergang vom kooperativen kohärenten, zum selbst-referentiellen Verhalten

wechselwirkender Teilchen, in diesem Falle von Elektronen, experimentell und theoretisch zu belegen.

\section{Literatur}

[1] B. Zimmermann et al., Nature Physics 2008, 4, 649

[2] D. Rolles et al., Nature 2005, 437, 711 ; U. Becker, Physik in unserer Zeit 2006, 37 (1), 7.

Uwe Becker, Fritz-Haber-Institut der MPG, Berlin.

\section{ABB. 1 ELEKTRONENINTERFERENZ}

Zwei Elektronen (rot und blau) werden kohärent aus einer spiegelsymmetrischen Anordnung zweier Atome herauskatapultiert. a) Ohne weitere Wechselwirkungen überlagern sich die beiden Elektronenwellen. b) Ist die Materiewellenlänge kürzer als der Abstand der beiden Atome, so erfährt das Elektron durch die Streuung eine Impulsänderung, die so groß ist, dass es über die Heisenbergsche Unschärferelation seinen Ursprungsort erfährt. Nun kann es sich mit seiner eigenen Welle, die es durch die Streuung kennt, selbst überlagern (Selbstinterferenz). c) Die Frequenz der Überlagerungswelle ist doppelt so groß wie die einzelnen Elektronen, da das Elektron zwischen seinem Entstehungsort und dem Nachbaratom einmal hin- und herlaufen muss. 